\title{
SOME INEQUALITIES OF LINEAR COMBINATIONS OF INDEPENDENT RANDOM VARIABLES. I.
}

\author{
MAOCHAO XU, ${ }^{*}$ Illinois State University \\ TAIZHONG HU, ${ }^{* *}$ University of Science and Technology of China
}

\begin{abstract}
In this paper we provide some sufficient conditions to stochastically compare linear combinations of independent random variables. The main results extend those given in Proschan (1965), Ma (1998), Zhao et al. (2011), and Yu (2011). In particular, we propose a new sufficient condition to compare the peakedness of linear combinations of independent random variables which may have heavy-tailed properties.
\end{abstract}

Keywords: Likelihood ratio order; majorization; peakedness order; stochastic order

2010 Mathematics Subject Classification: Primary 60E15

\section{Introduction}

Proschan (1965) showed that

$$
\mathrm{P}\left(\left|\sum_{i=1}^{n} \lambda_{i} X_{i}\right| \leq \varepsilon\right)
$$

is Schur concave in $\lambda=\left(\lambda_{1}, \lambda_{2}, \ldots, \lambda_{n}\right) \in \mathbb{R}_{+}^{n}$ for any $\varepsilon>0$ and random samples $X_{1}, X_{2}, \ldots$, $X_{n}$ from the same symmetric log-concave density population. That is,

$$
\left(\lambda_{1}, \lambda_{2}, \ldots, \lambda_{n}\right) \geq\left(\lambda_{1}^{\prime}, \lambda_{2}^{\prime}, \ldots, \lambda_{n}^{\prime}\right) \quad \Longrightarrow \quad \mathrm{P}\left(\left|\sum_{i=1}^{n} \lambda_{i} X_{i}\right| \geq \varepsilon\right) \geq \mathrm{P}\left(\left|\sum_{i=1}^{n} \lambda_{i}^{\prime} X_{i}\right| \geq \varepsilon\right),
$$

where ' $\stackrel{m}{\succeq}$ ' denotes the majorization order (see Marshall et al. (2011)). This result and its extensions have found wide applications in statistics, reliability theory, economic theory, mathematical evolutionary theory, and other fields. We refer the reader to Tong (1994), Jensen (1997), Ma (1998), Ibragimov (2007), and the references therein.

It is remarkable that Ibragimov (2007) made a significant contribution to this topic by showing that Proschan's result continues to hold in the case of convolutions of $\alpha$-symmetric distributions with $\alpha>1$, which can be used to model the heavy-tailedness phenomena. It should be noted that if $X$ has a log-concave density then its density has at most an exponential tail, i.e.

$$
f(x)=O(\exp (-\lambda x)), \quad \lambda>0, x \rightarrow \infty
$$

\footnotetext{
Received 7 January 2011; revision received 6 June 2011.

* Postal address: Department of Mathematics, Illinois State University, Normal, IL 61790-4520, USA.

Email address: mxu2@ilstu.edu

** Postal address: Department of Statistics and Finance, School of Management, University of Science and Technology of China, Hefei, Anhui 230026, P. R. China. Email address: thu@ustc.edu.cn

Supported by the NNSF of China (grant numbers 11071232 and 70821001) and the National Basic Research Program of China (973 Program, grant number 2007CB814901).
} 
(see Corollary 1 of An (1998)). See An (1998) and Bagnoli and Bergstrom (2005) for surveys of detailed properties of log-concave distributions.

The other significant contribution is by Yu (2011), who showed that

$$
\mathrm{P}\left(\sum_{i=1}^{n} \lambda_{i} X_{i} \leq t\right)
$$

is Schur concave in $\log \lambda=\left(\log \lambda_{1}, \log \lambda_{2}, \ldots, \log \lambda_{n}\right)$ for any $t \geq 0$ and positive, independent and identically distributed (i.i.d.) random samples $X_{1}, X_{2}, \ldots, X_{n}$ with $\log \left(X_{i}\right)$ having a $\log$ concave density. That is,

$$
\left(\log \lambda_{1}, \log \lambda_{2}, \ldots, \log \lambda_{n}\right) \stackrel{\mathrm{m}}{\succeq}\left(\log \lambda_{1}^{\prime}, \log \lambda_{2}^{\prime}, \ldots, \log \lambda_{n}^{\prime}\right) \Longrightarrow \sum_{i=1}^{n} \lambda_{i} X_{i} \geq_{\mathrm{st}} \sum_{i=1}^{n} \lambda_{i}^{\prime} X_{i}
$$

where $\lambda_{i}, \lambda_{i}^{\prime}>0$ for each $i$, and ' $\geq_{\text {st }}$ ' denotes the usual stochastic order.

In this paper we will further study this topic. First, we generalize Proschan's result from the i.i.d. case to the independent and possibly nonidentically distributed case under a weaker assumption. Then, we present the other sufficient condition which could be used to model heavytailed distributions, such as the Cauchy distribution, $t$-distribution, etc. We also generalize Yu's result to the independent and possibly nonidentically distributed case under a weaker assumption.

Pan et al. (2011), the companion paper to this one, unifies the study of linear combinations of independent random variables under the general setup by using some monotone transforms.

Throughout the paper, the terms 'increasing' and 'decreasing' are used to mean 'nondecreasing' and 'nonincreasing', respectively. A random variable $X$ is simply called symmetric if its probability density is symmetric about 0 .

\section{Preliminaries}

In this section we recall some stochastic orders and majorization orders, which will be used in the sequel.

Assume that the random variables $X$ and $Y$ have distribution functions $F$ and $G$, and density functions $f$ and $g$, respectively.

Definition 2.1. We say that $X$ is smaller than $Y$ in the usual stochastic order, denoted by $X \leq_{\text {st }} Y$, if $F(x) \geq G(x)$ for all $x$.

Definition 2.2. For two random variables $X$ and $Y$, and real constants $u$ and $v, X$ is said to be more peaked about $u$ than $Y$ about $v$, denoted by $X \geq{ }_{\text {peak }} Y$, if

$$
\mathrm{P}(|X-u| \geq t) \leq \mathrm{P}(|Y-v| \geq t), \quad t \geq 0 .
$$

When $u=v=0$, we simply say that $X$ is more peaked than $Y$, or, equivalently, $|X| \leq_{\text {st }}|Y|$.

Note that in the literature $X \leq{ }_{\text {peak }} Y$ if $X$ is more peaked about $u$ than $Y$ about $v$. In this paper we use the notation $X \geq_{\text {peak }} Y$ in Definition 2.2.

Definition 2.3. We say that $X$ is smaller than $Y$ in the likelihood ratio order, denoted by $X \leq \operatorname{lr} Y$, if $g(x) / f(x)$ is increasing in $x$ for which the ratio is well defined. 
For more discussions on stochastic orders, we refer the reader to the excellent book Shaked and Shanthikumar (2007).

We will also use the concept of majorization in our discussion. Let $x_{(1)} \leq x_{(2)} \leq \cdots \leq x_{(n)}$ be the increasing arrangement of components of the vector $\boldsymbol{x}=\left(x_{1}, x_{2}, \ldots, x_{n}\right)$.

Definition 2.4. For vectors $\boldsymbol{x}, \boldsymbol{y} \in \mathbb{R}^{n}, \boldsymbol{x}$ is said to be

- majorized by $\boldsymbol{y}$, denoted by $\boldsymbol{x} \stackrel{\mathrm{m}}{\preceq} \boldsymbol{y}$, if

$$
\sum_{i=1}^{j} x_{(i)} \geq \sum_{i=1}^{j} y_{(i)} \quad \text { for } j=1, \ldots, n-1,
$$

and $\sum_{i=1}^{n} x_{(i)}=\sum_{i=1}^{n} y_{(i)}$,

- weakly submajorized by $\boldsymbol{y}$, denoted by $\boldsymbol{x} \preceq_{\mathrm{w}} \boldsymbol{y}$, if

$$
\sum_{i=j}^{n} x_{(i)} \leq \sum_{i=j}^{n} y_{(i)} \quad \text { for } j=1, \ldots, n .
$$

For extensive and comprehensive details on the theory of majorization orders and their applications, see the book Marshall et al. (2011).

\section{Main results}

The following lemma, which is due to Birnbaum (1948) (see also Theorem 3.D.4 of Shaked and Shanthikumar (2007)), will be used in the sequel.

Lemma 3.1. Let $X_{1}, X_{2}, \ldots, X_{n}$ and $Y_{1}, Y_{2}, \ldots, Y_{n}$ be two sets of independent random variables, with the $X_{i} s$ and $Y_{i} s(i=1, \ldots, n)$ having distribution functions that are symmetric about possibly different centers and unimodal densities with possibly some probability mass at their respective centers. If $X_{i} \leq_{\text {peak }} Y_{i}$ for $i=1, \ldots, n$, then

$$
\sum_{i=1}^{n} X_{i} \leq \text { peak } \sum_{i=1}^{n} Y_{i}
$$

We first present the following useful lemma which is essentially due to Ma (1998).

Lemma 3.2. Let $X_{1}$ and $X_{2}$ be independent and symmetric random variables defined on $\mathbb{R}$ with densities $f_{1}$ and $f_{2}$, respectively. If $\left|X_{1}\right| \leq_{1 \mathrm{r}}\left|X_{2}\right|$ and $f_{2}(x)$ (or $\left.f_{1}(x)\right)$ is log-concave in $x \in \mathbb{R}$, then

$$
\left(\lambda_{1}, \lambda_{2}\right) \stackrel{\mathrm{m}}{\succeq}\left(\lambda_{1}^{\prime}, \lambda_{2}^{\prime}\right)
$$

implies that

$$
\mathrm{P}\left(\lambda_{(1)} X_{1}+\lambda_{(2)} X_{2} \geq t\right) \geq \mathrm{P}\left(\lambda_{(1)}^{\prime} X_{1}+\lambda_{(2)}^{\prime} X_{2} \geq t\right), \quad t \geq 0 .
$$

Now, we are ready to generalize Proschan's result under a weaker assumption.

Theorem 3.1. Let $X_{1}, X_{2}, \ldots, X_{n}$ be independent and symmetric random variables defined on $\mathbb{R}$ with densities $f_{1}, f_{2}, \ldots, f_{n}$, respectively. If $\left|X_{1}\right| \leq_{\operatorname{lr}}\left|X_{2}\right| \leq_{\operatorname{lr}} \cdots \leq_{\operatorname{lr}}\left|X_{n}\right|$ and $f_{i}(x)$ is log-concave for each $i$, then

$$
\left(\lambda_{1}, \ldots, \lambda_{n}\right) \succeq_{\mathrm{w}}\left(\lambda_{1}^{\prime}, \ldots, \lambda_{n}^{\prime}\right)
$$


implies that

$$
\mathrm{P}\left(\left|\sum_{i=1}^{n} \lambda_{(i)} X_{i}\right| \geq t\right) \geq \mathrm{P}\left(\left|\sum_{i=1}^{n} \lambda_{(i)}^{\prime} X_{i}\right| \geq t\right), \quad t \geq 0,
$$

or, equivalently,

$$
\sum_{i=1}^{n} \lambda_{(i)} X_{i} \leq \text { peak } \sum_{i=1}^{n} \lambda_{(i)}^{\prime} X_{i}
$$

Proof. According to Proposition 5.A.9 of Marshall et al. (2011, p. 177), if

$$
\left(\lambda_{(1)}, \ldots, \lambda_{(n)}\right) \succeq_{\mathrm{w}}\left(\lambda_{(1)}^{\prime}, \ldots, \lambda_{(n)}^{\prime}\right)
$$

then there exists a real vector $\left(\gamma_{1}, \ldots, \gamma_{n}\right)$ such that

$$
\left(\lambda_{(1)}, \ldots, \lambda_{(n)}\right) \stackrel{\mathrm{m}}{\geq}\left(\gamma_{(1)}, \ldots, \gamma_{(n)}\right) \geq\left(\lambda_{(1)}^{\prime}, \ldots, \lambda_{(n)}^{\prime}\right) .
$$

Since, for $\gamma_{(i)} \geq \lambda_{(i)}^{\prime}$

$$
\gamma_{(i)} X_{i} \leq_{\text {peak }} \lambda_{(i)}^{\prime} X_{i}
$$

using Lemma 3.1, it holds that

$$
\sum_{i=1}^{n} \gamma_{(i)} X_{i} \leq \text { peak } \sum_{i=1}^{n} \lambda_{(i)}^{\prime} X_{i}
$$

Hence, it is sufficient to prove that

$$
\sum_{i=1}^{n} \lambda_{(i)} X_{i} \leq \text { peak } \sum_{i=1}^{n} \gamma_{(i)} X_{i}
$$

Since the density of convolution of symmetric random variables with log-concave densities is symmetric and log-concave (see Ibragimov (1956) and also Dharmadhikari and Joag-Dev (1988)), by the nature of majorization, it is enough to verify that

$$
\lambda_{(1)} X_{1}+\lambda_{(2)} X_{2} \leq \text { peak } \gamma_{(1)} X_{1}+\gamma_{(2)} X_{2}
$$

From Lemma 3.2, it holds that, for $t \geq 0$,

$$
\mathrm{P}\left(\lambda_{(1)} X_{1}+\lambda_{(2)} X_{2} \geq t\right) \geq \mathrm{P}\left(\gamma_{(1)} X_{1}+\gamma_{(2)} X_{2} \geq t\right) .
$$

Using the symmetric properties of $X_{1}$ and $X_{2}$, it holds that, for $t \geq 0$,

$$
\mathrm{P}\left(\lambda_{(1)} X_{1}+\lambda_{(2)} X_{2} \leq-t\right)=\mathrm{P}\left(\lambda_{(1)} X_{1}+\lambda_{(2)} X_{2} \geq t\right) .
$$

So, it follows that

$$
\mathrm{P}\left(\lambda_{(1)} X_{1}+\lambda_{(2)} X_{2} \leq-t\right) \geq \mathrm{P}\left(\gamma_{(1)} X_{1}+\gamma_{(2)} X_{2} \leq-t\right) .
$$

Hence, (3.1) follows from (3.2) and (3.3). 
Remark 3.1. Ma (1998) proposed the following condition for Theorem 3.1:

$$
\left(\lambda_{1}, \ldots, \lambda_{n}\right) \stackrel{\mathrm{m}}{\succeq}\left(\lambda_{1}^{\prime}, \ldots, \lambda_{n}^{\prime}\right) .
$$

Zhao et al. (2011) presented another sufficient condition for the peakedness order, namely,

$$
\left(\log \lambda_{1}, \ldots, \log \lambda_{n}\right) \stackrel{m}{\succeq}\left(\log \lambda_{1}^{\prime}, \ldots, \log \lambda_{n}^{\prime}\right),
$$

and they also proposed the following condition for the i.i.d. case:

$$
\left(\log \lambda_{1}, \ldots, \log \lambda_{n}\right) \succeq_{\mathrm{w}}\left(\log \lambda_{1}^{\prime}, \ldots, \log \lambda_{n}^{\prime}\right) .
$$

However, by Theorem 5.A.2 of Marshall et al. (2011, p.167), it holds that

$$
\left(\log \lambda_{1}, \ldots, \log \lambda_{n}\right) \succeq_{\mathrm{w}}\left(\log \lambda_{1}^{\prime}, \ldots, \log \lambda_{n}^{\prime}\right) \quad \Longrightarrow \quad\left(\lambda_{1}, \ldots, \lambda_{n}\right) \succeq_{\mathrm{w}}\left(\lambda_{1}^{\prime}, \ldots, \lambda_{n}^{\prime}\right) .
$$

So, the condition presented in Theorem 3.1 is very general.

The following lemma presents another sufficient condition for symmetric distributions, which may have heavy-tailed properties.

Lemma 3.3. Let $X_{1}$ and $X_{2}$ be independent symmetric random variables defined on $\mathbb{R}$ with densities $f_{1}$ and $f_{2}$, respectively. If $\left|X_{1}\right| \leq_{\operatorname{lr}}\left|X_{2}\right|$ and $f_{2}\left(\mathrm{e}^{x}\right)\left(\right.$ or $\left.f_{1}\left(\mathrm{e}^{x}\right)\right)$ is log-concave in $x \in \mathbb{R}$ then

$$
\left(\log \lambda_{1}, \log \lambda_{2}\right) \stackrel{m}{\succeq}\left(\log \lambda_{1}^{\prime}, \log \lambda_{2}^{\prime}\right)
$$

implies that

$$
\mathrm{P}\left(\lambda_{(1)} X_{1}+\lambda_{(2)} X_{2} \leq t\right) \leq \mathrm{P}\left(\lambda_{(1)}^{\prime} X_{1}+\lambda_{(2)}^{\prime} X_{2} \leq t\right), \quad t \geq 0 .
$$

Proof. We only give the proof for the case that $f_{2}\left(\mathrm{e}^{x}\right)$ is log-concave; the proof of the other case is similar. For fixed $t>0$, define, for $\lambda \in(0,1]$,

$$
H_{\lambda}(t)=\mathrm{P}\left(\lambda X_{1}+\lambda^{-1} X_{2} \leq t\right)=\int_{-\infty}^{\infty} F_{2}\left(t \lambda-\lambda^{2} x\right) f_{1}(x) \mathrm{d} x,
$$

where $F_{i}$ is the distribution function of $X_{i}$ for $i=1,2$. It is sufficient to prove that $H_{\lambda}(t)$ is increasing in $\lambda \in(0,1]$. It is possible to justify differentiation under the integral sign, so

$$
H_{\lambda}^{\prime}(t)=\int_{-\infty}^{\infty}(t-2 \lambda x) f_{2}\left(t \lambda-\lambda^{2} x\right) f_{1}(x) \mathrm{d} x=\delta_{1}+\delta_{2}
$$

where

$$
\delta_{1}=\int_{0}^{t /(2 \lambda)}(t-2 \lambda x) f_{2}\left(t \lambda-\lambda^{2} x\right) f_{1}(x) \mathrm{d} x+\int_{t /(2 \lambda)}^{t / \lambda}(t-2 \lambda x) f_{2}\left(t \lambda-\lambda^{2} x\right) f_{1}(x) \mathrm{d} x
$$

and

$$
\delta_{2}=\int_{-\infty}^{0}(t-2 \lambda x) f_{2}\left(t \lambda-\lambda^{2} x\right) f_{1}(x) \mathrm{d} x+\int_{t / \lambda}^{\infty}(t-2 \lambda x) f_{2}\left(t \lambda-\lambda^{2} x\right) f_{1}(x) \mathrm{d} x .
$$

In the following, we will show that $\delta_{1} \geq 0$ and $\delta_{2} \geq 0$, separately. 
Proof of $\delta_{1} \geq 0$. For $\delta_{1}$, using the transform $x \mapsto t / \lambda-x$ in the second integral, it holds that

$$
\delta_{1}=\int_{0}^{t /(2 \lambda)}(t-2 \lambda x)\left[f_{2}\left(t \lambda-\lambda^{2} x\right) f_{1}(x)-f_{2}\left(\lambda^{2} x\right) f_{1}\left(\frac{t}{\lambda}-x\right)\right] \mathrm{d} x .
$$

It is enough to show that, for $0<x \leq t /(2 \lambda)$,

$$
f_{2}\left(t \lambda-\lambda^{2} x\right) f_{1}(x)-f_{2}\left(\lambda^{2} x\right) f_{1}\left(\frac{t}{\lambda}-x\right) \geq 0 .
$$

Since $\left|X_{1}\right| \leq \operatorname{lr}\left|X_{2}\right|$, it holds that, for $0<x \leq t /(2 \lambda)$,

$$
f_{2}\left(\frac{t}{\lambda}-x\right) f_{1}(x) \geq f_{1}\left(\frac{t}{\lambda}-x\right) f_{2}(x) .
$$

Thus, to prove (3.4), it suffices to prove that

$$
f_{2}\left(t \lambda-\lambda^{2} x\right) f_{2}(x) \geq f_{2}\left(\lambda^{2} x\right) f_{2}\left(\frac{t}{\lambda}-x\right) .
$$

Since, for $0<x \leq t /(2 \lambda)$ and $0<\lambda \leq 1$,

$$
\lambda^{2} x \leq \min \left\{t \lambda-\lambda^{2} x, x\right\}
$$

it holds that

$$
\left(\log \left(t \lambda-\lambda^{2} x\right), \log x\right) \stackrel{\mathrm{m}}{\preceq}\left(\log \left(\lambda^{2} x\right), \log \left(\frac{t}{\lambda}-x\right)\right)
$$

(see Yu (2011)). Since $f_{2}\left(\mathrm{e}^{x}\right)$ is log-concave, (3.5) holds. Therefore, $\delta_{1} \geq 0$.

Proof of $\delta_{2} \geq 0$. For $\delta_{2}$, using the transform $x \mapsto t / \lambda-x$ in the second integral, it holds that

$$
\delta_{2}=\int_{-\infty}^{0}(t-2 \lambda x)\left[f_{2}\left(t \lambda-\lambda^{2} x\right) f_{1}(x)-f_{2}\left(\lambda^{2} x\right) f_{1}\left(\frac{t}{\lambda}-x\right)\right] \mathrm{d} x .
$$

It is enough to show that, for $x \leq 0$,

$$
f_{2}\left(t \lambda-\lambda^{2} x\right) f_{1}(x)-f_{2}\left(\lambda^{2} x\right) f_{1}\left(\frac{t}{\lambda}-x\right) \geq 0 .
$$

Since $\left|X_{1}\right| \leq \operatorname{lr}\left|X_{2}\right|$ and $X_{1}$ and $X_{2}$ are symmetric, it holds that, for $t \geq 0$ and $x \leq 0$,

$$
f_{2}\left(\frac{t}{\lambda}-x\right) f_{1}(x) \geq f_{1}\left(\frac{t}{\lambda}-x\right) f_{2}(x) .
$$

Note that, for $x \leq 0,0<\lambda \leq 1$, and $t \geq 0$,

$$
-\lambda^{2} x \leq \min \left\{t \lambda-\lambda^{2} x,-x\right\}
$$

thus,

$$
\left(\log \left(t \lambda-\lambda^{2} x\right), \log (-x)\right) \stackrel{\mathrm{m}}{\preceq}\left(\log \left(-\lambda^{2} x\right), \log \left(\frac{t}{\lambda}-x\right)\right) .
$$

Since $f_{2}\left(\mathrm{e}^{x}\right)$ is log-concave, it follows that

$$
f_{2}\left(t \lambda-\lambda^{2} x\right) f_{2}(-x) \geq f_{2}\left(-\lambda^{2} x\right) f_{2}\left(\frac{t}{\lambda}-x\right),
$$


i.e.

$$
f_{2}\left(t \lambda-\lambda^{2} x\right) f_{2}(x) \geq f_{2}\left(\lambda^{2} x\right) f_{2}\left(\frac{t}{\lambda}-x\right) .
$$

Combining (3.7) and (3.6), it holds that $\delta_{2} \geq 0$.

This completes the proof.

Now, we show that Proschan's result will continue to be true for some heavy-tailed distributions. First, recall that, for a random variable with density function $f, f$ is said to be unimodal if there exists a value $a$ such that $f$ is increasing on $(-\infty, a]$ and decreasing on $[a,+\infty)$.

Theorem 3.2. Let $X_{1}, X_{2}, \ldots, X_{n}$ be independent symmetric random variables defined on $\mathbb{R}$ with unimodal densities $f_{1}, f_{2}, \ldots, f_{n}$, respectively. If $\left|X_{1}\right| \leq_{\operatorname{lr}}\left|X_{2}\right| \leq_{\operatorname{lr}} \cdots \leq_{\operatorname{lr}}\left|X_{n}\right|$, and $f_{i}\left(\mathrm{e}^{x}\right)$ is log-concave for each $i$, then

$$
\left(\log \lambda_{1}, \ldots, \log \lambda_{n}\right) \succeq_{\mathrm{w}}\left(\log \lambda_{1}^{\prime}, \ldots, \log \lambda_{n}^{\prime}\right)
$$

implies that

$$
\mathrm{P}\left(\left|\sum_{i=1}^{n} \lambda_{(i)} X_{i}\right| \geq t\right) \geq \mathrm{P}\left(\left|\sum_{i=1}^{n} \lambda_{(i)}^{\prime} X_{i}\right| \geq t\right), \quad t>0,
$$

or, equivalently,

$$
\sum_{i=1}^{n} \lambda_{(i)} X_{i} \leq_{\text {peak }} \sum_{i=1}^{n} \lambda_{(i)}^{\prime} X_{i}
$$

Proof. There exists a real vector $\left(\gamma_{1}, \ldots, \gamma_{n}\right)$ such that

$$
\left(\log \lambda_{(1)}, \ldots, \log \lambda_{(n)}\right) \stackrel{m}{\succeq}\left(\log \gamma_{(1)}, \ldots, \log \gamma_{(n)}\right) \geq\left(\log \lambda_{(1)}^{\prime}, \ldots, \log \lambda_{(n)}^{\prime}\right) .
$$

Since, for $\gamma_{(i)} \geq \lambda_{(i)}^{\prime}$,

$$
\gamma_{(i)} X_{i} \leq \text { peak } \lambda_{(i)}^{\prime} X_{i}
$$

and the $X_{i}$ s are unimodal, using Lemma 3.1, it holds that

$$
\sum_{i=1}^{n} \gamma_{(i)} X_{i} \leq \text { peak } \sum_{i=1}^{n} \lambda_{(i)}^{\prime} X_{i}
$$

Hence, it is sufficient to prove that

$$
\sum_{i=1}^{n} \lambda_{(i)} X_{i} \leq \text { peak } \sum_{i=1}^{n} \gamma_{(i)} X_{i}
$$

By the nature of majorization (see Lemma 2.B.1 of Marshall et al. (2011)), we assume without loss of generality that $\lambda_{(i)}=\gamma_{(i)}$ for $i=3, \ldots, n$ and

$$
\left(\log \lambda_{(1)}, \log \lambda_{(2)}\right) \stackrel{m}{\succeq}\left(\log \gamma_{(1)}, \log \gamma_{(2)}\right) .
$$

Since the convolution of two symmetric unimodal distributions on $\mathbb{R}$ is symmetric and unimodal (see Wintner (1938), and also Dharmadhikari and Joag-Dev (1988) and Purkayastha (1998)), by Lemma 3.1, it is enough to verify that

$$
\lambda_{(1)} X_{1}+\lambda_{(2)} X_{2} \leq \text { peak } \gamma_{(1)} X_{1}+\gamma_{(2)} X_{2}
$$


From Lemma 3.3, it holds that, for $t \geq 0$,

$$
\mathrm{P}\left(\lambda_{(1)} X_{1}+\lambda_{(2)} X_{2} \geq t\right) \geq \mathrm{P}\left(\gamma_{(1)} X_{1}+\gamma_{(2)} X_{2} \geq t\right) .
$$

Using the symmetric properties of $X_{1}$ and $X_{2}$ again, it holds that, for $t \geq 0$,

$$
\mathrm{P}\left(\lambda_{(1)} X_{1}+\lambda_{(2)} X_{2} \leq-t\right)=\mathrm{P}\left(\lambda_{(1)} X_{1}+\lambda_{(2)} X_{2} \geq t\right) .
$$

So, it follows that

$$
\mathrm{P}\left(\lambda_{(1)} X_{1}+\lambda_{(2)} X_{2} \leq-t\right) \geq \mathrm{P}\left(\gamma_{(1)} X_{1}+\gamma_{(2)} X_{2} \leq-t\right) .
$$

Hence, (3.8) follows from (3.9) and (3.10). This completes the proof.

Remark 3.2. (a) The condition that $f\left(\mathrm{e}^{x}\right)$ is log-concave in $x \in \mathbb{R}$ does not necessarily imply that the density function $f(x)$ is log-concave. For example, we may easily check that the $t$-distribution has the log-concave property of $f\left(\mathrm{e}^{x}\right)$. However, its density function is mixed.

(b) Theorem 3.2 can be used to construct the upper bound for some heavy-tailed distributions, such as the Cauchy distribution, $t$-distribution, etc., i.e.

$$
\mathrm{P}\left(\left|\sum_{i=1}^{n} \lambda_{i} X_{i}\right| \leq t\right) \leq \mathrm{P}\left(\left|\sum_{i=1}^{n} X_{i}\right| \leq \frac{t}{\tilde{\lambda}}\right), \quad t>0,
$$

where $\tilde{\lambda}=\left(\prod_{i=1}^{n} \lambda_{i}\right)^{1 / n}$, the geometric mean of $\lambda_{1}, \ldots, \lambda_{n}$.

In the following, we will extend Yu's result to independent and nonidentically distributed random variables. We first present the following lemma, which follows from the proof of $\delta_{1} \geq 0$ of Lemma 3.3.

Lemma 3.4. Let $X_{1}$ and $X_{2}$ be independent random variables defined on $\mathbb{R}_{+}$with densities $f_{1}$ and $f_{2}$, respectively. If $X_{1} \leq_{\operatorname{lr}} X_{2}$ and $f_{2}\left(\mathrm{e}^{x}\right)\left(\right.$ or $\left.f_{1}\left(\mathrm{e}^{x}\right)\right)$ is log-concave in $x \in \mathbb{R}$ then

$$
\left(\log \lambda_{1}, \log \lambda_{2}\right) \succeq\left(\operatorname{mog} \lambda_{1}^{\prime}, \log \lambda_{2}^{\prime}\right) \quad \Longrightarrow \quad \lambda_{(1)} X_{1}+\lambda_{(2)} X_{2} \geq_{\text {st }} \lambda_{(1)}^{\prime} X_{1}+\lambda_{(2)}^{\prime} X_{2} .
$$

Now, we are ready to present the following result.

Theorem 3.3. Let $X_{1}, X_{2}, \ldots, X_{n}$ be independent random variables defined on $\mathbb{R}_{+}$with densities $f_{1}, f_{2}, \ldots, f_{n}$, respectively. If $X_{1} \leq_{\operatorname{lr}} X_{2} \leq_{\operatorname{lr}} \cdots \leq_{\operatorname{lr}} X_{n}$, and $f_{i}\left(\mathrm{e}^{x}\right)$ is log-concave for each $i$, then

$$
\left(\log \lambda_{1}, \ldots, \log \lambda_{n}\right) \succeq_{\mathrm{w}}\left(\log \lambda_{1}^{\prime}, \ldots, \log \lambda_{n}^{\prime}\right) \Longrightarrow \sum_{i=1}^{n} \lambda_{(i)} X_{i} \geq_{\mathrm{st}} \sum_{i=1}^{n} \lambda_{(i)}^{\prime} X_{i}
$$

Proof. There exists a real vector $\left(\gamma_{1}, \ldots, \gamma_{n}\right)$ such that

$$
\left(\log \lambda_{(1)}, \ldots, \log \lambda_{(n)}\right) \stackrel{m}{\succeq}\left(\log \gamma_{(1)}, \ldots, \log \gamma_{(n)}\right) \geq\left(\log \lambda_{(1)}^{\prime}, \ldots, \log \lambda_{(n)}^{\prime}\right) .
$$

Since, for $\gamma_{(i)} \geq \lambda_{(i)}^{\prime}>0$,

$$
\gamma_{(i)} X_{i} \geq_{\mathrm{st}} \lambda_{(i)}^{\prime} X_{i}
$$


and the usual stochastic order is closed under convolution, it holds that

$$
\sum_{i=1}^{n} \gamma_{(i)} X_{i} \geq_{\mathrm{st}} \sum_{i=1}^{n} \lambda_{(i)}^{\prime} X_{i}
$$

Hence, it is sufficient to prove that

$$
\sum_{i=1}^{n} \lambda_{(i)} X_{i} \geq_{\mathrm{st}} \sum_{i=1}^{n} \gamma_{(i)} X_{i}
$$

By the nature of majorization, we only need to prove the case in which $n=2$, which follows from Lemma 3.4.

Remark 3.3. Theorem 3.3 can be applied to the following distributions (see also Yu (2011)).

(a) Uniform distribution with support $(0, s]$.

(b) Power function distribution with density function

$$
f(x)=c x^{c-1}, \quad 0<x \leq 1, c>0 .
$$

(c) Pareto distribution with density function

$$
f(x)=\beta x^{-\beta-1}, \quad x \geq 1, \beta>0 .
$$

(d) Beta distribution with density function

$$
f(x)=\frac{x^{\alpha-1}(1-x)^{\beta-1}}{B(\alpha, \beta)}, \quad 0<x<1, \alpha>0, \beta>1 .
$$

(e) F-distribution with density function

$$
f(x)=c x^{m_{1} / 2-1}\left[1+\frac{m_{1}}{m_{2}} x\right]^{-\left(m_{1}+m_{2}\right) / 2}, \quad x \geq 0,
$$

where $c>0$ depends on $\left(m_{1}, m_{2}\right)$ degrees of freedom.

(f) Generalized gamma distribution with density function

$$
f(x)=\frac{p}{\Gamma(q / p)} x^{q-1} \exp \left(-x^{p}\right), \quad x \geq 0, p, q>0 .
$$

When $p=q$, it reduces to the Weibull distribution; when $p=2$, it reduces to the generalized Rayleigh distribution; when $p=1$, it becomes the usual gamma distribution.

(g) Pareto distribution with density function

$$
f(x)=(a+1) b^{1+1 / a}(a x+b)^{-1 / a-2}, \quad x \geq 0, a>0, b>0 .
$$

Note that the distribution function is

$$
F(x)=1-\left(\frac{b}{a x+b}\right)^{1 / a+1}
$$


(h) Log-normal distribution with density function

$$
f(x)=\frac{1}{\sqrt{2 \pi} \sigma x} \exp \left(\frac{-(\log x-\mu)^{2}}{2 \sigma^{2}}\right), \quad x>0, \sigma>0 .
$$

(i) Burr distribution (or generalized log-logistic distribution) with density function

$$
f(x)=\frac{k c x^{c-1}}{\left(1+x^{c}\right)^{k+1}}, \quad x>0, c>0, k>0 .
$$

Some further examples can be found in Hu et al. (2004). Finally, it is worth pointing out that, for a positive random variable with decreasing density $f, f\left(\mathrm{e}^{x}\right)$ is log-concave on $\mathbb{R}$ if $f(x)$ is log-concave on $\mathbb{R}_{+}$(see Corollary 2 of An (1998)).

\section{References}

AN, M. Y. (1998). Logconcavity versus logconvexity: a complete characterization. J. Econom. Theory 80, 350-369.

Bagnoli, M. And Bergstrom, T. (2005). Log-concave probability and its applications. Econom. Theory 26, $445-469$.

Birnbaum, Z. W. (1948). On random variables with comparable peakedness. Ann. Math. Statist. 19, 76-81.

Dharmadhikari, S. and Joag-Dev, K. (1988). Unimodality, Convexity, and Applications. Academic Press, Boston, MA.

Hu, T., Nanda, A. K., Xie, H. And Zhu, Z. (2004). Properties of some stochastic orders: A unified study. Naval Res. Logistics 51, 193-216.

Ibragimov, I. A. (1956). On the composition of unimodal distributions. Theory Prob. Appl. 1, 255-280.

IBragimov, R. (2007). Efficiency of linear estimators under heavy-tailedness: convolutions of $\alpha$-symmetric distributions. Econometric Theory 23, 501-517.

Jensen, D. R. (1997). Peakedness of linear forms in ensembles and mixtures. Statist. Prob. Lett. 35, $277-282$.

MA, C. (1998). On peakedness of distributions of convex combinations. J. Statist. Planning Infer. 70, 51-56.

Marshall, A. W., Olkin, I. And Arnold, B. C. (2011). Inequalities: Theory of Majorization and Its Applications, 2nd edn. Springer, New York.

Pan, X., Xu, M. and Hu, T. (2011). Some inequalities of linear combinations of independent random variables: II. Tech. Rep., University of Science and Technology of China.

Proschan, F. (1965). Peakedness of distributions of convex combinations. Ann. Math. Statist. 36, 1703-1706.

Purkayastha, S. (1998). Simple proofs of two results on convolutions of unimodal distributions. Statist. Prob. Lett. 39, 97-100.

Shaked, M. And Shanthikumar, J. G. (2007). Stochastic Orders. Springer, New York.

ToNG, Y. L. (1994). Some recent developments on majorization inequalities in probability and statistics. Linear Algebra Appl. 199, 69-90.

Wintner, A. (1938). Asymptotic Distributions and Infinite Convolutions. Edwards Brothers, Ann Arbor, MI.

Yu, Y. (2011). Some stochastic inequalities for weighted sums. To appear in Bernoulli.

Zhao, P., Chan, P. S. ANd NG, H. K. T. (2011). Peakedness for weighted sums of symmetric random variables. J. Statist. Planning Infer. 141, 1737-1743. 